\title{
ARTICLE OPEN \\ Balanced translocation linked to psychiatric disorder, glutamate, and cortical structure/function
}

Pippa A Thomson ${ }^{1,10}$, Barbara Duff ${ }^{2,10}$, Douglas HR Blackwood ${ }^{2}$, Liana Romaniuk ${ }^{2}$, Andrew Watson ${ }^{2}$, Heather C Whalley ${ }^{2}$, Xiang Li $^{3}$, Maria R Dauvermann ${ }^{4}$, T William J Moorhead ${ }^{2}$, Catherine Bois ${ }^{2}$, Niamh M Ryan ${ }^{1}$, Holly Redpath ${ }^{2}$, Lynsey Hall ${ }^{2}$, Stewart W Morris ${ }^{1}$, Edwin JR van Beek ${ }^{3}$, Neil Roberts ${ }^{3}$, David J Porteous ${ }^{1}$, David St. Clair ${ }^{5}$, Brandon Whitcher ${ }^{6}$, John Dunlop ${ }^{7,8}$, Nicholas J Brandon ${ }^{7,8}$, Zoë A Hughes ${ }^{7}$, Jeremy Hall ${ }^{9}$, Andrew Mclntosh ${ }^{2}$ and Stephen M Lawrie ${ }^{2}$

Rare genetic variants of large effect can help elucidate the pathophysiology of brain disorders. Here we expand the clinical and genetic analyses of a family with a $(1 ; 11)(q 42 ; q 14.3)$ translocation multiply affected by major psychiatric illness and test the effect of the translocation on the structure and function of prefrontal, and temporal brain regions. The translocation showed significant linkage (LOD score 6.1) with a clinical phenotype that included schizophrenia, schizoaffective disorder, bipolar disorder, and recurrent major depressive disorder. Translocation carriers showed reduced cortical thickness in the left temporal lobe, which correlated with general psychopathology and positive psychotic symptom severity. They showed reduced gyrification in prefrontal cortex, which correlated with general psychopathology severity. Translocation carriers also showed significantly increased activation in the caudate nucleus on increasing verbal working memory load, as well as statistically significant reductions in the right dorsolateral prefrontal cortex glutamate concentrations. These findings confirm that the $t(1 ; 1)$ translocation is associated with a significantly increased risk of major psychiatric disorder and suggest a general vulnerability to psychopathology through altered cortical structure and function, and decreased glutamate levels.

npj Schizophrenia (2016) 2, Article number: 16024; doi:10.1038/npjschz.2016.24; published online 10 August 2016

\section{INTRODUCTION}

A balanced $t(1 ; 11)$ translocation was first described as a single locus major risk factor for major psychiatric disorder, including schizophrenia, bipolar disorder, and recurrent major depression, in a multiply affected Scottish pedigree with a maximum parametric LOD score of 7.1. ${ }^{1,2}$ The translocation breakpoint lies within the Disrupted in schizophrenia 1 (DISC1) and DISC1FP1/Boymaw genes. ${ }^{3}$ DISC1 encodes a multi-functional scaffold protein that mediates several processes that have been implicated in the etiology of major psychiatric disorders. Studies utilizing a variety of rodent models have also shown DISC1 to influence neurodevelopment, brain function, and behaviors thought to model schizophrenia and depression. ${ }^{4,5}$ DISC1 has been shown to mediate: neuronal proliferation, differentiation, migration, and integration, neuronal signaling and synaptic plasticity, ${ }^{4-6}$ regulation of neurogenesis and dendritic arborization, and the integration of cortical neurons. ${ }^{7-10}$ In addition, the expression of DISC1, DISC1FP/Boymaw, and the fusion protein caused by the translocation results in severe mitochondrial dysfunction. ${ }^{11-14}$ So far, there is no consistent evidence for a role for genetic variants in DISC1 in schizophrenia risk, including common variants in $D I S C 1,{ }^{15}$ and the potential role of DISC1 and 25 further candidate genes has been recently challenged. ${ }^{16,17}$ However, findings from the original
Scottish $t(1 ; 11)$ family have been presented that support the 'common disease; rare variant' hypothesis and suggest that DISC1 may have a role in major psychiatric disorders. ${ }^{18}$ The present study updates the linkage evidence for the $t(1 ; 11)$ translocation and risk of psychiatric disease and reports new neuroimaging measures from the family that provide evidence for biological consequences of the translocation.

We have previously reported that the P300 event-related potential measure of attention is consistently altered among $t(1 ; 11)$ carriers in a manner comparable to that seen in schizophrenia. ${ }^{2}$ Until now, we have not had the opportunity to test for the possible impact of the $t(1 ; 11)$ translocation on measures of brain structure, function, and metabolite concentrations. Several cognitive and clinical neuroimaging measures are highly heritable including measures of cortical thickness (CT), fractional anisotropy, and brain activation measures. ${ }^{19-21}$ The structure and function of the prefrontal cortex are implicated in a range of psychiatric disorders (such as schizophrenia, attentiondeficit/hyperactivity disorder, and autism); they have high levels of heritability and are commonly reported to be abnormal in unaffected relatives of patients. ${ }^{22}$ Studies examining the effects of common variants in DISC1 alleles in humans, although inconsistent, have suggested that variation at the DISC1 locus contributes

\footnotetext{
${ }^{1}$ Medical Genetics Section, Centre for Genomic and Experimental Medicine, University of Edinburgh, MRC Institute of Genetics and Molecular Medicine at the University of Edinburgh, Western General Hospital, Edinburgh, UK; ${ }^{2}$ Division of Psychiatry, Deanery of Clinical Sciences, University of Edinburgh, Royal Edinburgh Hospital, Morningside Park, Edinburgh, UK; ${ }^{3}$ Clinical Research Imaging Centre (CRIC), The Queen's Medical Research Institute, University of Edinburgh, UK; ${ }^{4}$ McGovern Institute for Brain Research, Massachusetts Institute of Technology, Cambridge, MA, USA; ${ }^{5}$ Institute of Medical Sciences, University of Aberdeen, Aberdeen, UK; ${ }^{6}$ Clinical \& Translational Imaging Group, Pfizer Global Research, Cambridge, MA, USA; ${ }^{7}$ Neuroscience Research Unit, Pfizer Global Research, Cambridge, MA, USA; ${ }^{8}$ AstraZeneca Neuroscience, Innovative Medicines and Early Development Biotech Unit, AstraZeneca, Cambridge, MA, USA and ${ }^{9}$ Neuroscience and Mental Health Research Institute, Cardiff University, Hadyn Ellis Building, Cardiff, UK. Correspondence: SM Lawrie (s.lawrie@ed.ac.uk)

${ }^{10}$ Joint first authors.

Received 18 April 2016; revised 30 June 2016; accepted 1 July 2016
} 
to structural and functional changes across the brain, but particularly in prefrontal and temporal regions. ${ }^{5,23-25}$ DISC1 has also been shown to regulate the healthy functioning of $\mathrm{N}$-methylD-aspartate receptors (NMDARs). ${ }^{26-28}$ A transgenic mouse model expressing a truncated form of Disc1, reflecting the effect of the translocation on the Disc1 protein, shows reduced corticalhippocampal connectivity, reduced $\mathrm{CT}$, and dysfunction of the glutamate system including reduced expression of NMDAR subunits in the hippocampus. ${ }^{10,29}$

Recent studies have suggested that the genetic risk associated with alterations in glutamatergic function may be implicated in the pathophysiological pathways of major psychiatric disorders. The glutamate hypothesis of schizophrenia is based on the NMDAR hypofunction model. ${ }^{30}$ Importantly, the glutamate hypothesis encompasses, rather than negates, the dopamine hypothesis of schizophrenia ${ }^{31-33}$ and is a possible final common interaction pathway for genetic risk factors for schizophrenia. ${ }^{34}$ The $\mathrm{N}$-methyl-Daspartate subtype of the glutamate receptor is implicated across anatomical, cellular, neurochemical, and neuronal levels in the development of schizophrenia, ${ }^{35}$ and the glutamate hypothesis provides arguably the best available account of the positive, negative, and cognitive symptoms seen in schizophrenia. Glutamatergic disruption has also been implicated in bipolar disorder and major depression. ${ }^{36,37}$ Furthermore, it is increasingly clear that major psychiatric disorders such as schizophrenia, bipolar disorder, and major depression share at least some genetic risk factors. ${ }^{38}$

Convergent findings supporting glutamatergic models have been reported from preclinical and clinical studies of the role of NMDARs during working memory (WM) coping and WM impairment after NMDAR antagonist treatment. ${ }^{39-43}$ Functional magnetic resonance imaging (fMRI) studies in humans have presented evidence for ketamine-induced effects on both WM and prefrontal region activation. ${ }^{39,40}$ The role of dorsolateral prefrontal cortex (DLPFC) dysfunction in WM deficits has been related to dopaminergic alterations in schizophrenia, bipolar disorder, and depression, but also to glutamatergic alterations and more specifically to dopamine-glutamate interactions. ${ }^{31,42,44}$ Magnetic resonance spectroscopy (MRS) provides a means to measure glutamate concentrations in circumscribed regions in vivo in humans. MRS studies have demonstrated alterations of glutamatergic concentrations in prefrontal regions in bipolar disorder and depression as well as schizophrenia. ${ }^{37}$

The primary aims of this study were to re-visit the multiply affected family segregating the $t(1 ; 11)$ translocation, generate non-parametric LOD scores across diagnoses on the updated pedigree and to investigate the effect of the $t(1 ; 11)$ translocation on brain structure, brain metabolite concentrations and brain function by imaging of family members with and without the translocation.

\section{RESULTS}

Extension of the family increases the evidence of linkage between the translocation and major mental illness

We previously published significant linkage of the $t(1 ; 11)$ translocation to major mental illness in a single Scottish family. ${ }^{2}$ This translocation is a unique variant private to this family. We therefore sought to confirm this linkage through the recruitment of additional members of the family to the study and full clinical re-evaluation of all participants by two psychiatrists. Clinical re-evaluation using all available current and historic information was undertaken on the full family as reported in Blackwood et al. ${ }^{2}$ Forty-two participants who took part in the previous studies volunteered for the present study. An additional 25 participants took part for the first time including four who carry the translocation. Historical information was reviewed for the remaining individuals. The extended family comprised 107 individuals in six generations and translocation status was determined in all but one individual (for whom no DNA was available and translocation status could not be imputed). In total, 37 participants carried the $t(1 ; 11)$ translocation and 69 were non-carriers. Details of diagnoses are given in Table 1. Two-point variance component analyses were performed using the program SOLAR (Table 2). To facilitate the

\begin{tabular}{|c|c|c|c|c|c|c|c|}
\hline Linkage analysis & $107^{\mathrm{a}}$ & 37 & 69 & $\begin{array}{l}\text { SCZ (2), SCZAFF (4), BP1 (2), } \\
\text { rMDD (8), MDD (4), } \\
\text { cyclothymia (3), conduct } \\
\text { disorder (3), generalized } \\
\text { anxiety (3), no psychiatric } \\
\text { disorder (2), inadequate } \\
\text { information (6) }\end{array}$ & $\begin{array}{l}\text { rMDD (3), MDD (3), generalized } \\
\text { anxiety (1), alcohol dependency } \\
\text { (1), no psychiatric } \\
\text { disorder (54), inadequate } \\
\text { information ( } 7 \text { ) }\end{array}$ & NA & NA \\
\hline $\begin{array}{l}\text { Clinical } \\
\text { assessment }\end{array}$ & 39 & 14 & 25 & $\begin{array}{l}\text { SCZ (1), SCZAFF (1), BP1 (1), } \\
\text { rMDD (3), MDD (3), cyclothymia } \\
\text { (3), conduct disorder (1), } \\
\text { no psychiatric disorder (1) }\end{array}$ & $\begin{array}{l}\text { rMDD (2), MDD (1), generalized } \\
\text { anxiety disorder (1) }\end{array}$ & $\begin{array}{l}\text { Sodium valproate (3), } \\
\text { +clozapine (1), } \\
\text { +risperidone (1), } \\
\text { +sertaline (1) }\end{array}$ & Amitriptyline (1) \\
\hline Structural MRI & 30 & 12 & 18 & $\begin{array}{l}\text { SCZ (1), schizoaffective (1), } \\
\text { BP1 (1) rMDD ( } 3 \text { ), MDD ( } 2), \\
\text { cyclothymia (3), conduct } \\
\text { disorder (1) }\end{array}$ & rMDD (2), MDD (1) & $\begin{array}{l}\text { Sodium valproate (3), } \\
\text { +clozapine (1), } \\
\text { +risperidone (1), } \\
\text { +sertaline (1) }\end{array}$ & Amitriptyline (1) \\
\hline Functional MRI & 23 & 8 & 15 & $\begin{array}{l}\text { rMDD (2), MDD (2), cyclothymia } \\
\text { (3), conduct disorder (1) }\end{array}$ & MDD (1) & None & Amitriptyline (1) \\
\hline
\end{tabular}


comparison of these results with those of the $t(1 ; 11)$ family as reported in Blackwood et al., ${ }^{2}$ variance component LOD scores were also generated on the previously published pedigree. In the extended family, the $t(1 ; 11)$ translocation was significantly linked to a phenotype that includes: only schizophrenia and schizoaffective disorder ( $\mathrm{LOD}=3.3$ ); only affective disorders (bipolar disorder

\begin{tabular}{|c|c|c|c|c|c|}
\hline \multirow[t]{2}{*}{ Model } & \multirow[t]{2}{*}{ Diagnoses } & \multicolumn{3}{|c|}{$\mathrm{N}$} & \multirow[t]{2}{*}{$\angle O D$} \\
\hline & & $\begin{array}{l}t(1 ; 11) \\
\text { carriers }\end{array}$ & $\begin{array}{c}t(1 ; 11) \\
\text { non-carriers }\end{array}$ & $A L L$ & \\
\hline \multicolumn{6}{|l|}{ Original family } \\
\hline Model 1 & SCZ, SCZAFF & 7 & 0 & 7 & 1.7 \\
\hline Model 2 & BP1, rMDD & 12 & 0 & 12 & 2.2 \\
\hline \multirow[t]{4}{*}{ Model 3} & $\begin{array}{l}\text { SCZ, SCZAFF, } \\
\text { BP1, rMDD }\end{array}$ & 19 & 0 & 19 & 3.8 \\
\hline & Unaffected & 7 & 21 & 28 & \\
\hline & Unknown & 2 & 1 & 3 & \\
\hline & Total & 30 & 28 & 58 & \\
\hline \multicolumn{6}{|l|}{ Extended family } \\
\hline Model 1 & SCZ, SCZAFF & 6 & 0 & 6 & 3.3 \\
\hline Model 2 & BP1, rMDD & 10 & 3 & 13 & 3.5 \\
\hline Model 3 & $\begin{array}{l}\text { SCZ, SCZAFF, } \\
\text { BP1, rMDD }\end{array}$ & 16 & 3 & 19 & 6.1 \\
\hline \multirow[t]{4}{*}{$\begin{array}{l}\text { Model } 3 \\
\text { +cyclothymia }\end{array}$} & $\begin{array}{l}\text { SCZ, SCZAFF, } \\
\text { BP1, rMDD, } \\
\text { cyclothymia }\end{array}$ & 19 & 3 & 22 & 7.9 \\
\hline & Unaffected & 2 & 53 & 55 & \\
\hline & Unknown & 8 & 8 & 16 & \\
\hline & Total & 37 & 70 & 107 & \\
\hline
\end{tabular}

Abbreviations: BP1, bipolar 1; MDD, single episode depression; $r M D D$ recurrent major depressive disorder; SCZ, schizophrenia; SCZAFF, schizoaffective disorder.

For comparison, the original family (Blackwood et al..$^{2}$ ) and the extended family two-point LOD scores are shown. Bold $=$ LOD scores $>3$. and recurrent major depression) $(\mathrm{LOD}=3.5)$; or all cases of major mental illness (schizophrenia, schizoaffective disorder, bipolar disorder, and recurrent major depressive disorder; LOD $=6.1$ ). A maximum LOD score was obtained if the phenotype is further extended to include three cases with cyclothymia ( $L O D=7.9$ ).

$t(1 ; 11)$ translocation carriers show localized differences in CT and gyrification index

Brain structural abnormalities, that have been identified between individuals with schizophrenia and unaffected individuals, are associated with both large genomic rearrangements and singlenucleotide polymorphisms including those in DISC1. ${ }^{45-50}$ We sought to identify the effects of the translocation on CT and cortical folding. Useable structural MRI, data were acquired for $12 \mathrm{t}(1 ; 11)$ translocation carriers and 18 non-carriers (Table 1). The average CT \pm s.d. was $2.40 \pm 0.16 \mathrm{~mm}$ for non-carriers and $2.23 \pm 0.12 \mathrm{~mm}$ for translocation carriers. The difference in CT between the two groups was statistically significant $(F(1,26)=4.248$, $P=0.049$ ), with greater $C T$ in non-carriers compared with carriers. The average local gyrification index (LGI) \pm s.d.: $2.94 \pm 0.19$ for noncarriers and $2.78 \pm 0.13$ for carriers. The one-way analysis of covariance, covarying for age and sex, found statistically significant differences of $L G I$ between groups $(F(1,28)=6.558$, $P=0.016)$. These results were, however, not robust after controlling for reduced intra-familial relatedness (global CT $P=0.055$ and LGI $P=0.23$ ). No significant differences were found in the global cortex surface area or estimated intracranial volume $(P>0.05)$.

Localized differences, many bilateral, in CT and LGI were found between $t(1 ; 11)$ translocation carriers and non-carriers on controlling for age and sex, and multiple comparisons (Figure 1), but only reduced left superior temporal sulcus (STS) CT in the temporal lobe and reduced right superior frontal sulcus LGI in the DLPFC were robust to controlling for intra-familial relatedness $(P=0.022$ and $P=0.025$, respectively).

All subgroups of $t(1 ; 11)$ translocation carriers had similarly low left STS CT and right superior frontal LGI values (Supplementary Results). LGI results for these regions split by translocation carrier diagnosis (psychosis, recurrent depression, and other) are shown in Supplementary Figure 1.
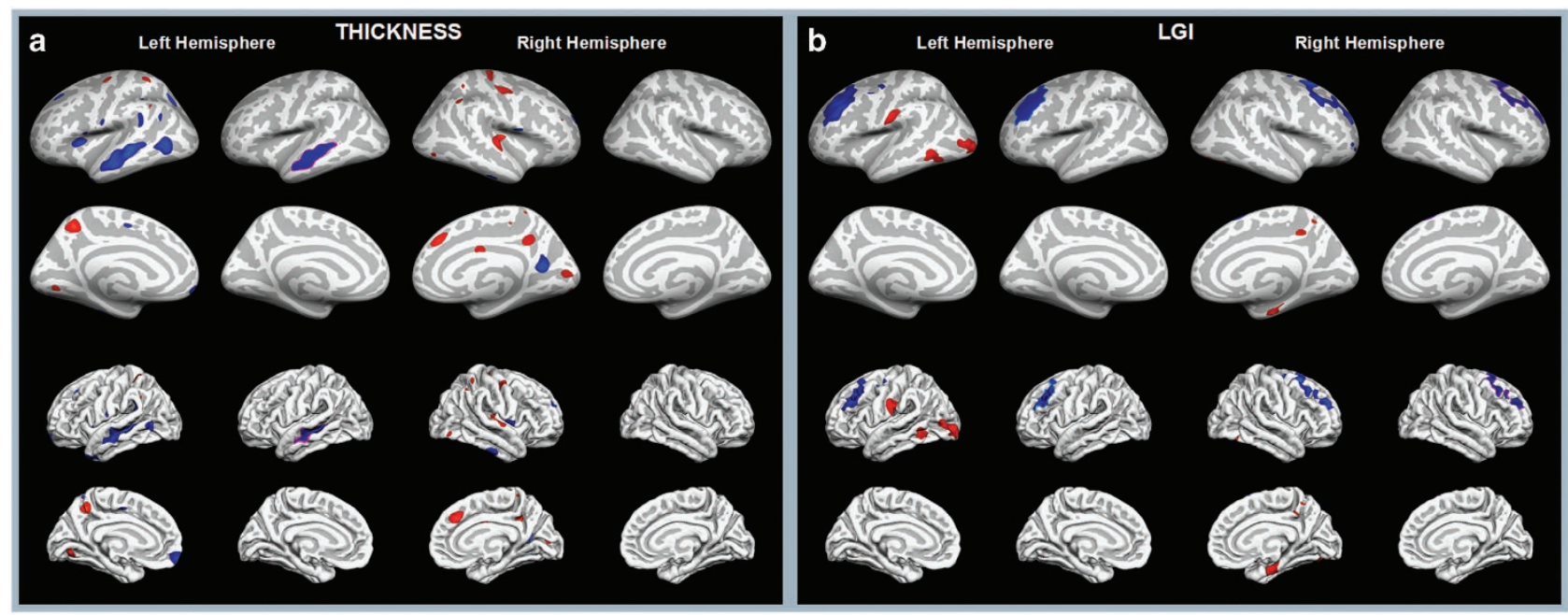

Figure 1. Effect of the translocation on cortical thickness and local gyrification index. (a) Cortical thickness and (b) local gyrification difference between translocation carriers and non-carriers rendered on the inflated and non-inflated cortical surface of the left and right brain templates. Columns 1, 3, 5, and 7 show the significance map of the difference; columns 2, 4, 6 , and 8 show the regions that survive the cluster-wise multiple comparisons correction $(P<0.05)$. The blue color indicates that the cortex is thinner and less gyrified for the translocation carriers compared with non-carriers, whereas the red color indicates the opposite effect. All these analyses are controlled for age and sex. Note however that only left superior temporal sulcus cortical thickness and right superior frontal sulcus gyrification index are robust to controlling for intra-familial relatedness (see text). LGl, local gyrification index. 
We examined the association between these structural measures and the contemporaneous mental state assessments with the Positive and Negative symptom scale (PANSS) in 30 subjects (both carriers and non-carriers). PANSS general psychopathology scores were negatively correlated with the left STS CT $(r=-0.43$, $P=0.016)$ and also with right superior frontal LGI $(r=-0.41$ $(P=0.025)$. PANSS-positive scores were negatively correlated with left STS CT $(r=-0.36, P=0.048)$, but not with the right superior frontal LGI $(r=-0.22, P=0.23)$. This suggests that the structural deficits in these regions effect symptomology, possibly through an impact on social cognition.

$t(1 ; 11)$ translocation carriers show increased activation in the caudate nucleus on increasing verbal WM load

Analyses of $\mathrm{fMRI}$ blood oxygen level-dependent activation profiles enable the in vivo study of brain activity during specific cognitive tasks. Patients with schizophrenia have been shown to have overactivation of brain regions during WM tasks, ${ }^{51}$ although reduced activation in contrast to healthy controls has also been reported. ${ }^{52}$ We sought evidence of an effect of the translocation on brain activation with increasing WM load. Useable fMRI data were acquired for eight family members with the $t(1 ; 11)$ carriers and 15 non-carriers (Table 1). These data were collected after the structural MRI and MRS acquisitions. Useable fMRI data were acquired for eight family members with the $t(1 ; 11)$ carriers and 15 non-carriers (Table 1).

Reaction time in the verbal WM 'N-back' task showed a trend for a main effect of WM load $(F(2,36)=2.571, P=0.09)$, but no significant effect of the group $(F(1,18)=0.018, P=0.89)$, or the group $\times$ WM load interaction $(F(2,36)=1.537, P=0.23)$. The sensitivity index (d) was computed to assess the behavioral performance between the groups. We found neither a significant main effect of WM load $(F(2,36)=1.24, P=0.30)$ nor group $(F(1,18)=0.930, P=0.35) \quad$ Furthermore, the group $\times W M$ load interaction was not significant $(F(2,36)=0.22, P=0.80)$.

When evaluating the effect of increasing WM load on blood oxygen level-dependent activation across translocation carriers and non-carriers, significant activation was found in bilateral inferior, middle and superior frontal cortices, bilateral inferior parietal lobules, right cerebellum, left inferior temporal gyrus, and the left middle orbital gyrus $(P<0.05$, Figure 2$)$. No regions demonstrated statistically significant group differences $(P>0.05)$. There was a significant group $\times$ WM load interaction in left caudate nucleus, with greater activation in translocation carriers with increasing load from $0-, 1-$, to 2-back (Figure 3; $F(1,17.5)=24.95, P<0.001)$, which was robust to controlling for familial relatedness $(P=0.001)$. Within the translocation carriers, caudate WM load response did not correlate with PANSS symptom measures $(P>0.05)$. The overactivation of the left caudate nucleus in $t(1: 11)$ translocation carriers may indicate inefficient corticostriatal information processing which is important in movement control, mood, and higher cognitive function.

$t(1 ; 11)$ translocation carriers show reduced levels of glutamate in the right dorsolateral prefrontal cortex

Metabolites reflecting glutamate neurotransmission have been shown, in some studies, to differ between patients and controls. ${ }^{31,42}$ We investigated the effect of the translocation on glutamate and $\mathrm{N}$-acetylaspartate (NAA) concentrations in prefrontal regions using MRS. Levels of glutamate and NAA were measured in the bilateral DLPFC and anterior cingulate cortex (ACC) of translocation carriers and non-carriers. The results are reported separately for each region.

In the right DLPFC, translocation carriers had significantly lower levels of glutamate than non-carriers ( $N=12$ carriers vs. $N=16$ non-carriers, mean glutamate levels \pm s.d.: $7.72 \pm 1.6$ vs. $9.86 \pm$ $2.4 \mathrm{mmol} / \mathrm{l}$ respectively, $P=0.021$ ). The diagnosis subgroups within translocation carriers had similarly low levels of glutamate (psychosis $(N=3) 6.9 \mathrm{mmol} / \mathrm{l}$, recurrent $\mathrm{MDD}(N=3) 7.4 \mathrm{mmol} / \mathrm{l}$, and others $(N=6) 7.7 \mathrm{mmol} / \mathrm{l})$. Correlations between glutamate levels and PANSS general psychopathology and positive symptom scales were non-significant $(P>0.4)$. In the left DLPFC, there was no significant effect of carrying the translocation on glutamate levels $(7.7 \pm 1.1$ vs. $8.3 \pm 1.7 \mathrm{mmol} / \mathrm{l}$, respectively, $P=0.42)$. Left DLPFC NAA levels were significantly lower in the translocation carriers $(N=11$ carriers vs. $N=16$ non-carriers, mean NAA levels $\pm \mathrm{s}$. d. was $11.9 \pm 2.1$ and $13.7 \pm 1.8 \mathrm{mmol} / \mathrm{l}$, respectively, $P=0.025$ ), although these were not robust to controlling for reduced intrafamilial relatedness. NAA levels were not significantly lower in the right DLPFC ( $N=12$ carriers vs. $N=16$ non-carriers, mean NAA levels \pm s.d. was $13.1 \pm 2.5$ and $14.3 \pm 1.6 \mathrm{mmol} / \mathrm{l}$, respectively, $P=0.17$ ).

There were no significant differences between translocation carriers and non-carriers in the concentrations of glutamate or NAA in the ACC $(P>0.05)$.

These data suggest a specific glutamate deficit in the DLPFC of $t(1 ; 11)$ translocation carriers, consistent with a direct genetic effect.

\section{DISCUSSION}

The current study provides a contemporary and extensive followup to earlier work on the $t(1 ; 11)$ translocation and confirms that it is a rare variant of large effect, linked with genome-wide significance to a broad psychiatric phenotype that includes schizophrenia, bipolar disorder, and recurrent major depression and, shown here for the first time, is associated with structural and functional changes to the brain detected by neuroimaging.

Specifically, carriers of the $t(1 ; 11)$ translocation demonstrate reductions in glutamate concentrations in the right DLPFC consistent with the glutamate hypothesis, as well as reduced CT and gyral folding, and increased left caudate nucleus blood oxygen level-dependent activation when compared with noncarriers. Of these, only the localized CT and gyrification reductions were associated with general psychopathology and only the left STS CT reductions were associated with positive symptom severity in $\mathrm{t}(1 ; 11)$ translocation carriers. Elsewhere, we have shown widespread reductions in white matter tract integrity in $t(1 ; 11)$ translocation carriers. ${ }^{53}$ These striking effects were observed in what are relatively small comparative studies that benefit from being part of a long-term within-family study of the incidence and evolution of psychiatric illness, controlled for by the presence or absence of the $t(1 ; 11)$ translocation. The within-family design optimizes the genetic matching over the genome. However, the correspondingly low numbers may have limited our ability to detect some effects of the translocation, and correlations between these effects and symptom correlations, but did not obscure the impact of the $t(1 ; 11)$ translocation on aspects of human brain structure, function, and neurochemistry.

The $t(1 ; 11)$ translocation was strongly associated with a range of psychiatric outcomes in the family, but with more homogeneous effects on imaging measures. This pattern of results, of a greater genetic impact on neurobiological measures than clinical phenotypes, is consistent with the previous P300 event-related potential study of the $t(1 ; 11)$ family, which found abnormalities in those with the translocation regardless of clinical diagnosis. ${ }^{2}$ Comparable effects of major psychiatric illness on human brain imaging measures, ${ }^{54-56}$ as well as a reduction in DLPFC glutamate concentration, ${ }^{37}$ have been noted in multiple case-control studies, transcending conventional diagnostic boundaries. The pattern of neuroimaging abnormalities detected in this study is striking and reflective of the pattern identified in a mouse model of the translocation in which a truncated Disc 1 fragment is expressed in a single copy (Disc1tr hemizygous, Hemi, mice). ${ }^{10}$ Similarly to the family, this mouse model shows both cortical thinning and deficits in the glutamate system, ${ }^{10,29}$ suggesting that these deficits, at 


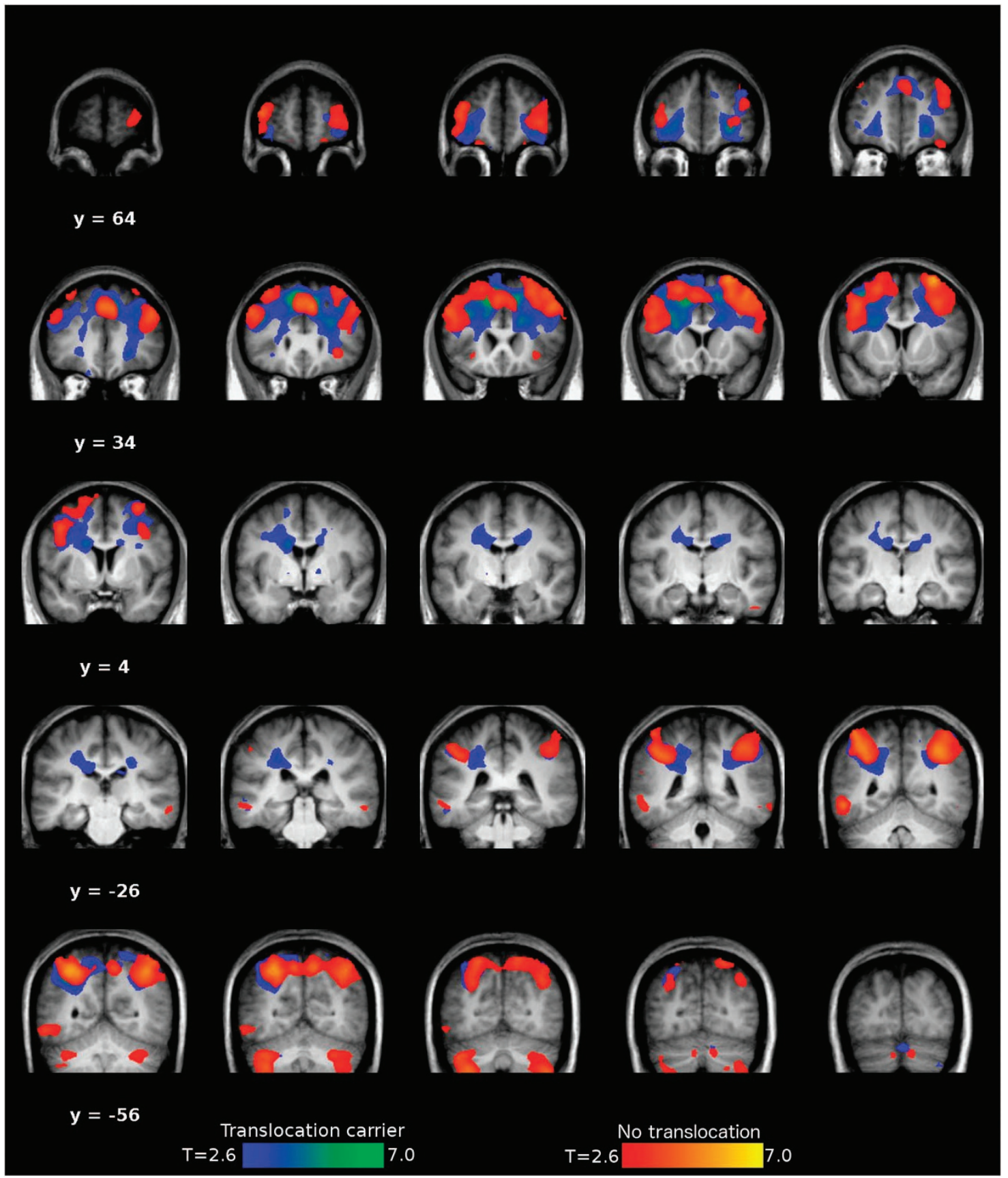

Figure 2. Effect of increasing working memory load on blood oxygen level-dependent activity measures in translocation carriers and noncarriers. Coronal sections through the brain to show the effects of increasing working memory load (from 0- to 1-to 2-back) in the N-back task on functional MRI in $\mathrm{t}(1 ; 11)$ translocation carriers and non-carriers. The image is thresholded at $P<0.001$, uncorrected, to show regional activations. These were statistically significant in/across both groups in bilateral inferior, middle, and superior frontal cortices, bilateral inferior parietal lobules, right cerebellum, left inferior temporal gyrus, and the left middle orbital gyrus at $P<0.05$, family-wise error corrected for multiple comparisons. There were no statistically significant group differences at a family-wise error-corrected threshold of $P<0.05$.

least in part, may be the direct result of disruption of the DISC1 locus.

We found relatively few correlations between the neuroimaging measures and symptom severity in participants, nor any apparent instances where the effects of the $t(1 ; 11)$ translocation could be accounted for by extreme results in one or more diagnostic subgroups (Supplementary Data). This supports the interpretation that the brain imaging abnormalities evident in the $t(1 ; 11)$ carriers are primarily genetic in origin and confer risk across a range of phenotypes. The abnormalities in $\mathrm{CT}$ are in keeping with the generalized reductions in $\mathrm{CT}$ reported in many psychiatric disorders, e.g., Hulshoff Pol et al. ${ }^{57}$ Additional structural genetic variants, polygenic risk factor loads, and environmental risk factors may then mediate the development of schizophrenia, bipolar disorder, and recurrent depression. In this respect, longitudinal, within-family studies can be a powerful complement to large case-control studies for which clinical heterogeneity may obscure underlying commonalities.

In conclusion, our results substantiate prior evidence for a genome-wide significant effect of the $t(1 ; 11)$ translocation on 
a

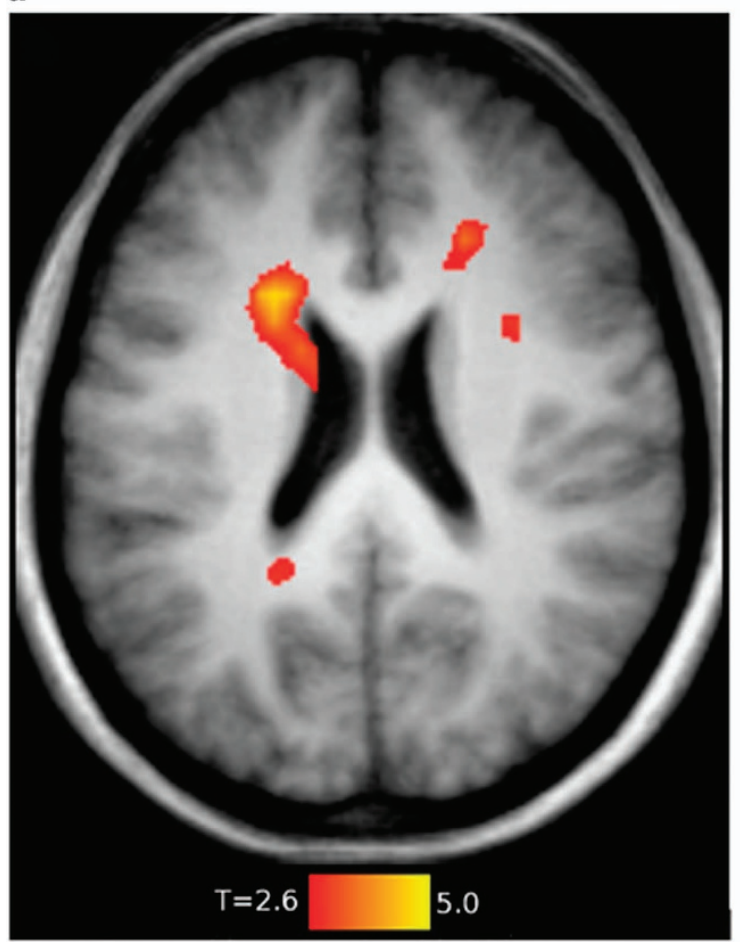

b

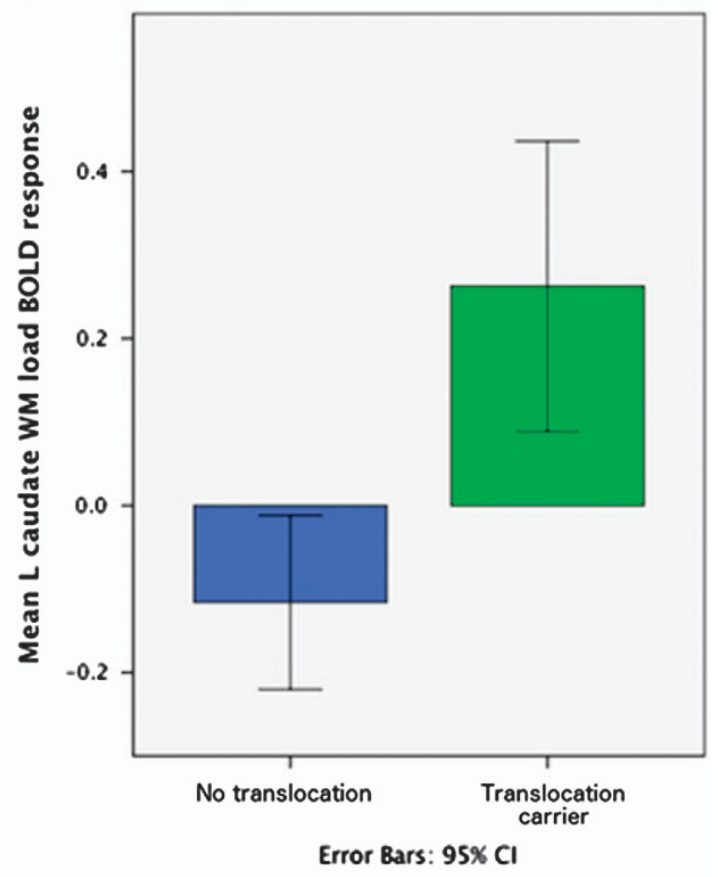

Figure 3. Differential brain activation during increasing working memory load between translocation carriers and non-carriers. The effects of increasing working memory load (from 0 - to 1 - to 2-back) in the N-back task on functional MRI comparing t(1;11) translocation carriers and non-carriers, controlling for age and sex. (a) Transverse slice $(z=22)$ displaying the statistically significant group $\times$ load interaction in left caudate, a family-wise error-corrected $P<0.05$. (b) Contrast estimates in the left caudate for 2 -back $>0$-back for $\mathrm{t}(1 ; 11)$ carriers and noncarriers to indicate the size of the effect.

cross-disorder risk of major mental illness. The idea that study of rare genetic variants can highlight biological mechanisms of relevance is not new, indeed is widely accepted and acknowledged as providing valuable insights, as, e.g., the single-gene risk factors for dementia (APP, PSEN1, and 2), and the role of copynumber variants and de novo mutations of high penetrance in schizophrenia and autism spectrum disorder. ${ }^{58-60}$ The $t(1 ; 11)$ translocation, similarly, provides a clear-cut and useful biological model for major psychiatric disorder. The most parsimonious explanation is that the molecular mechanism is explained by disruption of genes on chromosomes 1 and 11, including the DISC1 gene; a mechanism supported by the wealth of evidence linking DISC1 biology to independently constructed core concepts in psychopathology. As such, our findings suggest translational opportunities for experimental studies on carefully phenotyped and genetically analyzed subjects that may generalize to the wider population to speed the discovery and evaluation of much needed evidence based interventions.

\section{MATERIALS AND METHODS}

\section{Participants}

Individuals with and without the $t(1 ; 11)$ translocation were recruited from a previously reported extended Scottish family. ${ }^{1,261}$ Some of the family had been in contact with members of the research team for many years and through them other members of the family were invited to participate. None of the family members who participated suffered from substance dependence or neurological injury or illness or had MRI safety preclusions. A summary of the number of individual participants in each study, their translocation status, and medication at the time of each study is given in Table 1.

\section{Clinical and cognitive assessment}

Psychiatric diagnosis according to DSM-IV (TR) criteria was established by consensus between two trained psychiatrists (D.B. and A.W., one of whom (A.W.) was blind to the individuals karyotype status). Diagnostic information was obtained by a face-to-face semi-structured interview using the Structured Clinical Interview for DSM-IV (SCID) ${ }^{62}$ supplemented by reviews of hospital records and collateral information from hospital psychiatrists and general practitioners. At the time of interview, the following ratings were completed: PANSS, ${ }^{63}$ Scale for the Assessment of Negative Symptoms; ${ }^{64}$ Global Assessment of Function; ${ }^{65}$ Young Mania Rating scale; $^{66}$ and the Hamilton Depression Rating Scale. ${ }^{67}$ Current and premorbid IQ were assessed using the National Adult Reading Test ${ }^{68}$ and the Wechsler Abbreviated Scale of Intelligence (WASI). ${ }^{69}$ Historic information reported by StClair et al. ${ }^{1}$ and Blackwood et al. ${ }^{2}$ was retained and reviewed for subjects who were deceased or not available for followup. The operational criteria symptom check-list ${ }^{70}$ was completed based on psychiatric case notes and interview data. Sample demographics are provided in Table 3.

\section{Genotyping of the translocation}

The $t(1 ; 11)$ translocation status of family members was originally ascertained by karyotyping as reported by Jacobs et al., ${ }^{61}$ and subsequently by StClair et al. ${ }^{1}$ and Blackwood et al. ${ }^{2}$ For the current study, a PCR assay specific for the $t(1 ; 11)$ breakpoint was devised, validated on 22 samples from participants for whom karyotype status was known (including 14 carriers) and used to determine the presence or absence of the $t(1 ; 11)$ in 25 new participants (Supplementary Methods). DNA samples were available for PCR-based verification of translocation status from 48 individuals (18 carriers and 30 non-carriers). Translocation status was imputed in additional family members were possible.

\section{Multimodal neuroimaging}

Neuroimaging measures were: (i) global and local CT, estimated intracranial volume, surface area, and gyrification from index structural 
Table 3. Sample demographics for the $t(1 ; 11)$ translocation carriers and non-carriers

\begin{tabular}{|c|c|c|c|c|}
\hline & \multicolumn{2}{|c|}{$\begin{array}{l}t(1 ; 11) \\
\text { carriers }\end{array}$} & \multicolumn{2}{|c|}{$\begin{array}{c}t(1 ; 11) \\
\text { non-carriers }\end{array}$} \\
\hline Sample size & \multicolumn{2}{|c|}{14} & \multicolumn{2}{|c|}{25} \\
\hline Gender & & & & \\
\hline Male & \multicolumn{2}{|c|}{8} & \multicolumn{2}{|c|}{12} \\
\hline Female & \multicolumn{2}{|c|}{6} & \multicolumn{2}{|c|}{13} \\
\hline & Mean & s.d. & Mean & s.d. \\
\hline Age & 55.64 & 15.28 & 38.6 & 20.14 \\
\hline Premorbid $\mathrm{IQ}^{\mathrm{a}}$ & 103.07 & 7.23 & 104.54 & 6.36 \\
\hline Current $\mathrm{IQ}^{\mathrm{a}}$ & 88.15 & 16.79 & 93.47 & 10.68 \\
\hline \multicolumn{5}{|l|}{ PANSS } \\
\hline Total score & 46.86 & 24.38 & 32.84 & 4.82 \\
\hline Negative symptoms & 9.64 & 9.06 & 7 & 0 \\
\hline Positive symptoms & 10.14 & 6.29 & 7 & 0 \\
\hline General Symptoms & 27.07 & 10.73 & 18.84 & 4.82 \\
\hline SANS & 7.07 & 26.46 & 0.24 & 1.2 \\
\hline GAF & 75.14 & 22.41 & 88.2 & 12.82 \\
\hline YMRS & 2.93 & 5.4 & NA & NA \\
\hline HRSD & 5.57 & 6.21 & 2.8 & 5.43 \\
\hline \multicolumn{5}{|c|}{$\begin{array}{l}\text { Abbreviations: GAF, Global Assessment of Function; HRSD, Hamilton } \\
\text { Depression Rating Scale; PANSS, Positive and Negative Symptoms Scale; } \\
\text { SANS, Scale for the Assessment of Negative Symptoms; WASI, Wechsler } \\
\text { Abbreviated Scale of Intelligence; YMRS, Young Mania Rating Scale. } \\
\text { Premorbid IQ (National Adult Reading Test) and current IQ (WASI). } \\
\text { a } N=13 \text { in the translocation carriers and } N=15 \text { in the non-carrier groups. }\end{array}$} \\
\hline
\end{tabular}

MRI, (ii) brain activation during the verbal WM 'N-back' task using functional MRI, and (iii) glutamate and NAA levels in the DLPFC and ACC using MRS. Detail of the pre-processing and analysis of the neuroimaging measures are given in Supplementary Methods. Specific details for each imaging modality are included in the results section. Hypothesis testing was undertaken with significance set at $P<0.05$ after correction for multiple comparisons. We further investigated whether any differences between the groups were associated with psychopathology by examining the results for diagnostic subgroups (psychosis, recurrent depression, and others) of those with the translocation, as well as relating imaging measures to the PANSS general and positive psychotic symptom severity ratings in those with and without the $t(1 ; 11)$ translocation.

\section{Statistics}

Two-point variance component linkage analyses of the translocation status with SCID diagnosis were performed using SOLAR software package ${ }^{71}$ under the assumption of the liability threshold model for discrete traits. ${ }^{72,73}$ LOD scores were adjusted for deviation of the phenotype from normality, by correcting the inflation of the observed LOD scores, by the comparison with LOD scores generated using a simulated normally distributed trait with 10,000 permutations, using the lodadj command in SOLAR. ${ }^{74}$ These adjusted LOD scores are presented in the results.

All neuroimaging data group contrasts were conducted controlling for age and sex, and for intra-familial relatedness. Intra-familial relatedness, how related individuals are within the family, was modeled by creating an inverse relationship matrix using pedigree kinship information. Where univariate models of the imaging measures, controlling for age and sex, were nominally significant $(P<0.05)$, the analyses were repeated using mixed linear models implemented in ASReml-R (www.vsni.co.uk/software/ asreml), fitting the inverse relationship matrix as a random effect, allowing us to control for familial structure. The significance of fixed effects within the model was then assessed using a conditional Wald F-test.

\section{Study approval}

The study was approved by the Multicentre Research Ethics Committee for Scotland (09/MRE00/81). A detailed description of the study was given and written informed consent was obtained from all individuals before participation.

\section{ACKNOWLEDGMENTS}

This work was supported by an award (NS-EU-166) to Stephen Lawrie and colleagues from the Translational Medicine Research Collaboration-a consortium made up of the Universities of Aberdeen, Dundee, Edinburgh, and Glasgow, the four associated NHS Health Boards (Grampian, Tayside, Lothian, and Greater Glasgow \& Clyde), Scottish Enterprise, and Pfizer (formerly Wyeth). We would like to acknowledge the contributions of the following Wyeth/Pfizer colleagues over the course of the project: Menelas Pangalos, Sharon Rosenzweig-Lipson, Garry Honey, Phil Murphy, Tim McCarthy, Bill Vennart, and Michael Ehlers. The scans were acquired at the Clinical Research Imaging Centre (http://www.cric.ed.ac.uk). We would like to thank Scott Semple (MRI physicist), Annette Cooper, and her team of radiographers at CRIC for help in organizing the study and acquiring the scans. The investigators also acknowledge the support of National Health Service Research Scotland, through the Scottish Mental Health Research Network (www.smhrn.org.uk), particularly James McKirdy, who provided assistance with subject recruitment. We would also like to thank Karine MacRitchie for help in recruiting and interviewing some participants. During this work, T.W.J.M. and M.R.D. received support from the Dr Mortimer and Theresa Sackler Foundation, H.C.W. was supported by a Dorothy Hodgkin fellowship from the Royal Society and a JMAS SIM fellowship from the Royal College of Physicians of Edinburgh, and J.H. and A.M. were supported by CSO Scottish Senior Clinical Research Fellowships. P.A.T. was supported by a RCUK fellowship. N.M.R. is supported by NIH award R01MH102088 to D.J.P. E.J.R.v.B. and N.R. were supported by the Scottish Imaging Network-a Platform for Scientific Excellence (www.sinapse.ac. uk). Above all, we would like to thank the participants and their families for their participation.

\section{CONTRIBUTIONS}

Study design: S.M.L., J.H., A.M., D.H.R.B., N.J.B., J.D., and Z.A.H.; data analysis: M.R.D. T.W.J.M., P.A.T., B.D., D.H.B., L.R., A.W., H.C.W., L.X., E.J.R.v.B., N.R., C.B., N.M.R., H.R., L.H., and S.W.M.; manuscript preparation: S.M.L., P.A.T., A.W., and B.D.; additional editing: M.R.D., E.J.R.v.B., N.R., and D.J.P. All authors commented on drafts of the paper.

\section{COMPETING INTERESTS}

S.M.L. has received financial support for research from Roche and Abbvie in relation to therapeutic studies of people with schizophrenia. He has also received personal payment for input into educational initiatives from Roche, Janssen, and Sunovion. These received funds do not present a conflict of interest with the present study. B.W., J.D., N.J.B., and Z.A.H. are all current or former employees of Pfizer. J.D. and N.J.B. are current employees of AstraZeneca. The remaining authors declare no conflict of interest.

\section{REFERENCES}

1. St Clair, D. et al. Association within a family of a balanced autosomal translocation with major mental illness. Lancet 336, 13-16 (1990).

2. Blackwood, D. H. et al. Schizophrenia and affective disorders--cosegregation with a translocation at chromosome $1 \mathrm{q} 42$ that directly disrupts brain-expressed genes: clinical and P300 findings in a family. Am. J. Hum. Genet. 69, 428-433 (2001).

3. Millar, J. K. et al. Disruption of two novel genes by a translocation co-segregating with schizophrenia. Hum. Mol. Genet. 9, 1415-1423 (2000).

4. Brandon, N. J. \& Sawa, A. Linking neurodevelopmental and synaptic theories of mental illness through DISC1. Nat. Rev. Neurosci. 12, 707-722 (2011).

5. Thomson, P. A. et al. DISC1 genetics, biology and psychiatric illness. Front. Biol. (Beijing) 8, 1-31 (2013)

6. Chubb, J. E., Bradshaw, N. J., Soares, D. C., Porteous, D. J. \& Millar, J. K. The DISC locus in psychiatric illness. Mol. Psychiatry 13, 36-64 (2008).

7. Singh, K. K. et al. Common DISC1 polymorphisms disrupt Wnt/GSK3beta signaling and brain development. Neuron 72, 545-558 (2011).

8. Duan, X. et al. Disrupted-In-Schizophrenia 1 regulates integration of newly generated neurons in the adult brain. Cell 130, 1146-1158 (2007).

9. Hikida, T. et al. Dominant-negative DISC1 transgenic mice display schizophreniaassociated phenotypes detected by measures translatable to humans. Proc. Natl Acad. Sci. USA 104, 14501-14506 (2007)

10. Shen, S. et al. Schizophrenia-related neural and behavioral phenotypes in transgenic mice expressing truncated Disc1. J. Neurosci. 28, 10893-10904 (2008). 
11. Eykelenboom, J. E. et al. A $\mathrm{t}(1 ; 11)$ translocation linked to schizophrenia and affective disorders gives rise to aberrant chimeric DISC1 transcripts that encode structurally altered, deleterious mitochondrial proteins. Hum. Mol. Genet. 21, 3374-3386 (2012).

12. Ji, B. et al. Inhibition of protein translation by the DISC1-Boymaw fusion gene from a Scottish family with major psychiatric disorders. Hum. Mol. Genet 23, 5683-5705 (2014).

13. Ji, B., Kim, M., Higa, K. K. \& Zhou, X. Boymaw, overexpressed in brains with major psychiatric disorders, may encode a small protein to inhibit mitochondrial function and protein translation. Am. J. Med. Genet. B Neuropsychiatr. Genet. 168B, 284-295 (2015)

14. Zhou, X., Chen, Q., Schaukowitch, K., Kelsoe, J. R. \& Geyer, M. A. Insoluble DISC1Boymaw fusion proteins generated by DISC1 translocation. Mol. Psychiatry 15, 669-672 (2010).

15. Schizophrenia Working Group of the Psychiatric Genomics C. Biological insights from 108 schizophrenia-associated genetic loci. Nature 511, 421-427 (2014).

16. Sullivan, P. F. Questions about DISC1 as a genetic risk factor for schizophrenia. Mol. Psychiatry 18, 1050-1052 (2013).

17. Farrell, M. S. et al. Evaluating historical candidate genes for schizophrenia. Mol. Psychiatry 20, 555-562 (2015).

18. Porteous, D. J. et al. DISC1 as a genetic risk factor for schizophrenia and related major mental illness: response to Sullivan. Mol. Psychiatry 19, 141-143 (2014).

19. Blokland, G. A., de Zubicaray, G. I., McMahon, K. L. \& Wright, M. J. Genetic and environmental influences on neuroimaging phenotypes: a meta-analytical perspective on twin imaging studies. Twin Res. Hum. Genet. 15, 351-371 (2012).

20. Glahn, D. C., Thompson, P. M. \& Blangero, J. Neuroimaging endophenotypes: strategies for finding genes influencing brain structure and function. Hum. Brain Mapp. 28, 488-501 (2007).

21. Kochunov, P. et al. Heritability of fractional anisotropy in human white matter: A comparison of Human Connectome Project and ENIGMA-DTI data. Neuroimage 111, 300-311 (2015)

22. Lawrie, S. M., McIntosh, A. M., Hall, J., Owens, D. G. \& Johnstone, E. C. Brain structure and function changes during the development of schizophrenia: the evidence from studies of subjects at increased genetic risk. Schizophr. Bull. 34, 330-340 (2008)

23. Callicott, J. H. et al. Variation in DISC1 affects hippocampal structure and function and increases risk for schizophrenia. Proc. Natl Acad. Sci. USA 102, 8627-8632 (2005).

24. Cannon, T. D. et al. Association of DISC1/TRAX haplotypes with schizophrenia, reduced prefrontal gray matter, and impaired short- and long-term memory. Arch. Gen. Psychiatry 62, 1205-1213 (2005).

25. Duff, B. J., Macritchie, K. A., Moorhead, T. W., Lawrie, S. M. \& Blackwood, D. H. Human brain imaging studies of DISC1 in schizophrenia, bipolar disorder and depression: a systematic review. Schizophr. Res. 147, 1-13 (2013).

26. Hayashi-Takagi, A. et al. Disrupted-in-Schizophrenia 1 (DISC1) regulates spines of the glutamate synapse via Rac1. Nat. Neurosci. 13, 327-332 (2010).

27. Wei, J. et al. Regulation of N-methyl-D-aspartate receptors by disrupted-inschizophrenia-1. Biol. Psychiatry 75, 414-424 (2014).

28. Wang, G. \& Zhu, J. J. DISC1 dynamically regulates synaptic N-methyl-D-aspartate responses in excitatory neurons. Biol. Psychiatry. 75, 348-350 (2014).

29. Dawson, N. et al. Altered functional brain network connectivity and glutamate system function in transgenic mice expressing truncated Disrupted-inSchizophrenia 1. Transl. Psychiatry 5, e569 (2015).

30. Olney, J. W. \& Farber, N. B. Glutamate receptor dysfunction and schizophrenia. Arch. Gen. Psychiatry 52, 998-1007 (1995).

31. Coyle, J. T. Glutamate and schizophrenia: beyond the dopamine hypothesis. Cell. Mol. Neurobiol. 26, 365-384 (2006).

32. Lisman, J. E. et al. Circuit-based framework for understanding neurotransmitter and risk gene interactions in schizophrenia. Trends Neurosci. 31, 234-242 (2008).

33. Merritt, K., Egerton, A., Kempton, M. J., Taylor, M. J. \& McGuire, P. K. Nature of Glutamate Alterations in Schizophrenia: A Meta-analysis of Proton Magnetic Resonance Spectroscopy Studies. JAMA Psychiatry 73, 665-674 (2016).

34. Hall, J. et al. Associative learning and the genetics of schizophrenia. Trends Neurosci. 32, 359-365 (2009).

35. Coyle, J. T. NMDA receptor and schizophrenia: a brief history. Schizophr. Bull. $\mathbf{3 8}$, 920-926 (2012).

36. Sanacora, G., Treccani, G. \& Popoli, M. Towards a glutamate hypothesis of depression: an emerging frontier of neuropsychopharmacology for mood disorders. Neuropharmacology 62, 63-77 (2012).

37. Yuksel, C. \& Ongur, D. Magnetic resonance spectroscopy studies of glutamaterelated abnormalities in mood disorders. Biol. Psychiatry 68, 785-794 (2010).

38. Cross-Disorder Group of the Psychiatric Genomics C. Identification of risk loci with shared effects on five major psychiatric disorders: a genome-wide analysis. Lancet 381, 1371-1379 (2013).
39. Anticevic, A. et al. NMDA receptor function in large-scale anticorrelated neural systems with implications for cognition and schizophrenia. Proc. Natl Acad. Sci. USA 109, 16720-16725 (2012).

40. Driesen, N. R. et al. The impact of NMDA receptor blockade on human working memory-related prefrontal function and connectivity. Neuropsychopharmacology 38, 2613-2622 (2013).

41. Fitzgerald, P. J. The NMDA receptor may participate in widespread suppression of circuit level neural activity, in addition to a similarly prominent role in circuit level activation. Behav. Brain Res. 230, 291-298 (2012).

42. Javitt, D. C. Glutamate and schizophrenia: phencyclidine, N-methyl-D-aspartate receptors, and dopamine-glutamate interactions. Int. Rev. Neurobiol. 78, 69-108 (2007).

43. Moghaddam, B. \& Javitt, D. From revolution to evolution: the glutamate hypothesis of schizophrenia and its implication for treatment. Neuropsychopharmacology 37, 4-15 (2012).

44. Tanaka, S. Dopaminergic control of working memory and its relevance to schizophrenia: a circuit dynamics perspective. Neuroscience 139, 153-171 (2006).

45. Carless, M. A. et al. Impact of DISC1 variation on neuroanatomical and neurocognitive phenotypes. Mol. Psychiatry 16, 1096-1104, 1063 (2011).

46. Dauvermann, M. R. et al. Relationship between gyrification and functional connectivity of the prefrontal cortex in subjects at high genetic risk of schizophrenia. Curr. Pharm. Des. 18, 434-442 (2012).

47. Kahler, A. K. et al. Effect of DISC1 SNPs on brain structure in healthy controls and patients with a history of psychosis. Am. J. Med. Genet. B Neuropsychiatr. Genet. 159B, 722-730 (2012)

48. Nesvag, R. et al. Reduced brain cortical folding in schizophrenia revealed in two independent samples. Schizophr. Res. 152, 333-338 (2014).

49. Schmitt, J. E. et al. Aberrant Cortical Morphometry in the 22q11.2 Deletion Syndrome. Biol. Psychiatry 78, 135-143 (2015).

50. Wolthusen, R. P. et al. Genetic underpinnings of left superior temporal gyrus thickness in patients with schizophrenia. World J. Biol. Psychiatry 1-11 (2015); e-pub ahead of print.

51. Dauvermann, M. R. et al. Computational neuropsychiatry-schizophrenia as a cognitive brain network disorder. Front. Psychiatry 5, 30 (2014).

52. Jiang, S. et al. Cerebral inefficient activation in schizophrenia patients and their unaffected parents during the n-back working memory task: a family fMRI Study. PLoS One 10, e0135468 (2015).

53. Whalley, H. C. et al. Effects of a balanced translocation between chromosomes 1 and 11 disrupting the DISC1 locus on white matter integrity. PLoS One 10, e0130900 (2015).

54. Arnone, D. et al. Magnetic resonance imaging studies in bipolar disorder and schizophrenia: meta-analysis. Br. J. Psychiatry 195, 194-201 (2009).

55. Mclntosh, A. M. et al. White matter tractography in bipolar disorder and schizophrenia. Biol. Psychiatry 64, 1088-1092 (2008).

56. Whalley, H. C. et al. Review of functional magnetic resonance imaging studies comparing bipolar disorder and schizophrenia. Bipolar. Disord. 14, 411-431 (2012).

57. Hulshoff Pol, H. E. et al. Overlapping and segregating structural brain abnormalities in twins with schizophrenia or bipolar disorder. Arch. Gen. Psychiatry 69, 349-359 (2012).

58. Ripke, S. et al. Genome-wide association analysis identifies 13 new risk loci for schizophrenia. Nat. Genet. 45, 1150-1159 (2013).

59. Rovelet-Lecrux, A. et al. A genome-wide study reveals rare CNVs exclusive to extreme phenotypes of Alzheimer disease. Eur. J. Hum. Genet. 20, 613-617 (2012).

60. Cruchaga, C. et al. Rare variants in APP, PSEN1 and PSEN2 increase risk for AD in late-onset Alzheimer's disease families. PLoS One 7, e31039 (2012).

61. Jacobs, P. A. et al. Studies on a family with three cytogenetic markers. Ann. Hum. Genet. 33, 325-336 (1970).

62. First, M. B., Spitzer, R. L., Gibbon, M. \& Williams, J. B. Structured Clinical Interview for DSM-IV Axis I Disorders (SCID-I), Clinician Version, Administration Booklet. (American Psychiatric Publishing, 2012).

63. Kay, S. R., Fiszbein, A. \& Opler, L. A. The positive and negative syndrome scale (PANSS) for schizophrenia. Schizophr. Bull. 13, 261-276 (1987).

64. Andreasen, N. C. The Scale for the Assessment of Negative Symptoms (SANS): conceptual and theoretical foundations. Br. J. Psychiatry Suppl. 7, 49-58 (1989).

65. Pedersen, G. \& Karterud, S. The symptom and function dimensions of the Global Assessment of Functioning (GAF) scale. Compr. Psychiatry 53, 292-298 (2012).

66. Young, R. C., Biggs, J. T., Ziegler, V. E. \& Meyer, D. A. A rating scale for mania: reliability, validity and sensitivity. Br. J. Psychiatry 133, 429-435 (1978).

67. Hamilton, M. Rating depressive patients. J. Clin. Psychiatry 41, 21-24 (1980).

68. Nelson, H. E. \& Willison, J. R. The Revised National Adult Reading Test-Test Manual. (Vol NFER-Nelson. Windsor, UK, 1991).

69. Wechsler, D. \& Hsiao-pin, C. WASI-II: Wechsler Abbreviated Scale of Intelligence. (Pearson, 2011)

70. McGuffin, P., Farmer, A. \& Harvey, I. A polydiagnostic application of operational criteria in studies of psychotic illness. Development and reliability of the OPCRIT system. Arch. Gen. Psychiatry 48, 764-770 (1991). 
71. Almasy, L. \& Blangero, J. Multipoint quantitative-trait linkage analysis in general pedigrees. Am. J. Hum. Genet. 62, 1198-1211 (1998).

72. Duggirala, R., Williams, J. T., Williams-Blangero, S. \& Blangero, J. A variance component approach to dichotomous trait linkage analysis using a threshold model. Genet. Epidemiol. 14, 987-992 (1997).

73. Williams, J. T. \& Blangero, J. Power of variance component linkage analysis-II. Discrete traits. Ann. Hum. Genet. 68, 620-632 (2004).

74. Blangero, J., Williams, J. T. \& Almasy, L. Robust LOD scores for variance component-based linkage analysis. Genet. Epidemiol. 19 Suppl 1, S8-14 (2000).

Supplementary Information accompanies the paper on the npj Schizophrenia website (http://www.nature.com/npjschz) (c) (i) This work is licensed under a Creative Commons Attribution 4.0 International License. The images or other third party material in this article are included in the article's Creative Commons license, unless indicated otherwise in the credit line; if the material is not included under the Creative Commons license, users will need to obtain permission from the license holder to reproduce the material. To view a copy of this license, visit http://creativecommons.org/licenses/ by/4.0/

C) The Author(s) 2016 